\title{
SOME REMARKS ON CONNECTED SETS
}

\author{
P. ERDÖS
}

This note will consist of a few disconnected remarks on connected sets.

i. Swingle ${ }^{1}$ raised the question whether the plane is the sum of $c$ disjoint biconnected sets. The answer as we shall show is affirmative.

First we construct a biconnected set $A$ with a dispersion point $x$ such that any two points of $A-x$ can be separated. (The first such set was constructed by Wilder. ${ }^{2}$ Our construction will be very similar to that of Burton Jones. ${ }^{3}$ )

Our biconnected set will contain the origin $\mathfrak{D}$, at most one other point on lines through the origin with irrational slopes, and no point other than the origin on lines with rational slopes. Further it will contain at least one point on every cut of the plane. It is easy to see that such a set exists. Every cut of the plane contains a closed subset which also cuts the plane, and the power of closed sets is known to be $c$. Let us well order the closed cuts $C_{1}, C_{2}, \cdots, C_{\gamma}, \cdots, \gamma<\Omega_{\alpha}$, where $\Omega_{\alpha}$ is the least ordinal number of power $c$. We construct $A$ as follows: $\mathfrak{D}$ belongs to $A$. We shall choose a point $x_{\gamma}$ on $C_{\gamma}$ and we shall have $A=U_{x_{\gamma}}$. We shall determine $x_{\gamma}$ by transfinite induction. Suppose we have already determined $x_{\delta}, \delta<\gamma$, we determine $x_{\gamma}$ as follows: if $C_{\gamma}$ contains $\mathfrak{D}$ then $x_{\gamma}=\mathfrak{D}$. If $C_{\gamma}$ does not contain $\mathfrak{D}$ then clearly $C_{\gamma}$ has to intersect $c$ lines through $\mathfrak{D}$. Therefore we can find a point $x_{\gamma} \in C_{\gamma}$ such that $\left(\mathfrak{O}, x_{\gamma}\right)$ has irrational slope and does not go through any other point $x_{\delta}, \delta<\gamma$. (We denote by $(a, b)$ the line through the points $a$ and $b$.) This way we construct $A$. Clearly $A$ is biconnected. First of all $A$ is connected since it intersects every cut of the plane. Also any two points of $A-\mathfrak{D}$ can be separated since the two points $x_{1}$ and $x_{2}$, say, are on different irrational lines through the origin, and a rational line, which of course does not intersect $A-\mathfrak{D}$, will separate them. This completes the proof. The origin we call the center of $A$.

Now we shall split the plane into the sum of $c$ such disjoint sets $A_{\gamma}, \gamma<\Omega_{\alpha}, \Omega_{\alpha}$ the smallest ordinal of power $c$. Let us well order the points $x_{\gamma}$ of the plane and the closed cuts $C_{\gamma}$ of the plane. Our first step is to select $x_{1}$ as the center of $A_{1}$ and a suitable point of $A_{1}$ on $C_{1}$.

Received by the editors February 2, 1944.

1 P. M. Swingle, Amer. J. Math. vol. 54 (1932) p. 532.

2 R. L. Wilder, Bull. Amer. Math. Soc. vol. 33 (1927) p. 423.

${ }^{3}$ Burton Jones, Bull. Amer. Math. Soc. vol. 48 (1942) p. 115. 
(By suitable we mean that it does not lie on any line with rational slope through $x_{1 .}$ ) Our second step is to select the first point which has not yet been selected as center of $A_{2}$, then select suitable points on $C_{1}$ and $C_{2}$ for $A_{2}$ and on $C_{2}$ for $A_{1}$. Suppose that this construction has been already carried out for all $\delta<\gamma$. Then the $\gamma$ th step consists of the following construction: First we take the first $x_{k}$ which has not yet been chosen as center of $A_{\boldsymbol{\gamma}}$. Then we choose suitable points on all the $C_{\delta}, \delta \leqq \gamma$, for $A_{\gamma}$ and suitable points on $C_{\gamma}$ for all the $A_{\delta}, \delta<\gamma$. it is easy to see that this construction can be carried out since before the $\gamma$ th step we have chosen less than $c$ points $x_{j}$, and a cut $C_{\gamma}$ if it does not go through the origin intersects $c$ lines through the origin. (if the cut $C$ goes through the origin of one or more of the $A_{\gamma}$ 's then of course we can choose the origin.) Thus the construction can be carried out for all ordinals $\gamma<\Omega_{\alpha}$. Hence we get $c$ sets $A_{\gamma}$ where $\mathrm{U} A_{\gamma}$ is the whole plane, $A_{\gamma_{1}} \cap A_{\gamma_{2}}=0$ and the $A_{\gamma}$ are all biconnected, which completes the proof of the theorem.

A simple modification of this proof would give the following result: Let $m \leqq c$ be any cardinal number greater than 2 , then the plane can be expressed as the sum of $m$ disjoint biconnected sets. ${ }^{4}$

ii. Let $C$ be any connected set. Knaster and Kuratowskis proved that there exists a proper connected subset of $C$. (Single points, of course, do not count as connected sets.) Their proof is very simple. Let $p \in C$ be arbitrary. If $C-p$ is connected our result is proved. If $C-p$ is not connected it can be written in the form $U+V$ where $U$ and $V$ are separated. But then it is easy to see that both $U+p$ and $V+p$ are connected.

It seems likely that the following result is true: every connected set $C$ contains a connected subset $C^{\prime}$ such that $C-C^{\prime}$ has power $c$. I am unable to prove this. It is easy to see that if a set $C$ does not satisfy this conjecture it has to be both biconnected and widely connected, thus must be rather pathological.

A. Stone ${ }^{6}$ proved that every connected set $C$ has a connected subset $C^{\prime}$ such that $C-C^{\prime}$ is infinite. Proof: First we can assume that $C-p$ is connected for every $p \in C$, for if not then at least one of the sets $U+p$ or $V+p$ has a complement of power $c$. ( $U$ and $V$ were defined in the previous paragraph.) Similarly for every sequence $p_{1}, p_{2}, \cdots, p_{n}, C-p_{1}-p_{2}-\cdots-p_{n}$ is connected. Choose $p_{1}, p_{2}, \cdots$

4 Swingle proved (ibid.) that the Euclidean $n$ space is the sum of $r$ biconnected sets if $r$ is any finite number not less than $n+1$, also that it is the sum of $\aleph_{0}$ biconnected sets. He also showed that $n$-space is not the sum of $n$ or less biconnected sets.

5 Knaster-Kuratovski, Fund. Math. vol. 2 (1921) p. 206.

- Arthur Stone, oral communication. 
such that it will converge to a point $p \in C$. Then $C-\sum_{i=1}^{i} p_{i}$ is connected. For if $\sum_{i=1}^{\infty} p_{i}$ would separate $C$, a closed subset of it would also separate $C$. But a closed subset of $\sum_{i=1}^{\infty} p_{i}$ is finite, and so by a. sumption can not separate $C$, and this completes the proof.

In this connection it might be of some interest to construct a connected set $A$ with the following properties: (1) no finite subset disconnects $A,(2)$ every countable dense subset totally disconnects $A$. For our construction we shall have to use the continuum hypothesis.

First we need the following lemma: Let $S_{1}$ and $S_{2}$ be two countable disjoint sets. $S_{1}$ is everywhere dense in the plane. Then there exist countably many closed Jordan curves $J_{r}$ such that $J_{r} \cap S_{1}$ is dense on $J_{r}, J_{r} \cap S_{2}=0$, and to any two points $p$ and $q$ there exists an $r$ such that $J_{r}$ separates $p$ and $q$. The proof does not present any difficulties and can be omitted.

And now to our construction. ${ }^{7}$ First we well order the countable dense subsets of the plane, $D_{1}, D_{2}, \cdots, D_{\alpha}, \cdots$ (their number is clearly $c=\boldsymbol{N}_{1}$; the continuum hypothesis has been assumed). Also we well order all the closed connected cuts of the plane $C_{\delta}$, again clearly $\delta<\Omega_{1}$ (it is well known that every cut of the plane contains a closed connected cut). We shall now construct our set $A$ by transfinite induction. Let $\Delta$ be any given countable dense set, put $\Delta C A$. Our first step is to define a countable subset $P_{1}$ of $C_{1}$. If $\Delta \cap C_{1}$ is infinite we put $P_{1}=0$, if $C_{1} \cap \Delta$ is finite, we choose an arbitrary countably infinite $P_{1} \subset C_{1}$ and put $P_{1} \subset A$. Consider next the smallest $\alpha$ such that $D_{\alpha} C \Delta+P_{1}$. Put $S_{1}=D_{\alpha}$ and $S_{2}=\Delta+P_{1}-D_{\alpha}$. Then by our lemma there exists a countable collection of closed Jordan curves $J_{r}^{(1)}$ such that $J_{r}^{(1)} \cap D_{\alpha}$ is dense on $J_{r}^{(1)}$ and $J_{r}^{(1)} \cap \Delta+P_{1}-D_{\alpha}=0$ and any two points $p$ and $q$ are separated by some $J_{r}^{(1)}$. Now we make the condition that no point of any $P_{\beta}(\beta>1)$ should lie on any $J_{r}^{\beta}$. Suppose we carried out our construction for any $\gamma<\delta$, we shall show that we can carry it out for $\gamma=\delta$. Consider $C_{\delta}$; if $C_{\delta} \cap \Delta+\sum_{\beta<\delta} P_{\beta}$ is infinite, then $P_{\delta}=0$. If $C_{\delta} \cap \Delta+\sum_{\beta<\delta} P_{\beta}$ is finite, then we choòse a countably infinite subset $P_{\delta}$ of $C_{\delta}$, such that $P_{\delta}$ does not meet $\sum_{\lambda<\delta} \sum_{r-1}^{\infty} J_{r}^{\lambda}$. In other words $P_{\delta}$ does not meet any of countably many Jordan curves (we have assumed the continuum hypothesis). We have to prove that such a choice of $P_{\delta}$ is possible. By assumption $\left(\Delta+\sum_{\beta<\delta} P_{\beta}\right) \cap C_{\delta}$ is finite. For each of the Jordan curves $J_{r}^{\lambda}$ (with $\lambda<\delta$ ) $J_{r}^{\lambda} \cap \Delta+\sum_{\beta<\delta} P_{\beta}$ is dense on $J_{r}^{\lambda}$, therefore $C_{\delta} \cap J_{r}^{\lambda}$ is nowhere dense on $C_{\delta}$ (for if not, since $C_{\delta}$ is closed, it would contain a portion of $J_{r}^{\lambda}$ and therefore $C_{\delta} \cap\left(\Delta+\sum_{\beta<\delta} P_{\beta}\right)$ would be infinite, as $\Delta+\sum_{\beta<\delta} P_{\beta}$ is dense on $\left.J_{r}^{\lambda}\right)$.

7 Our construction will be similar to Miller's construction of a biconnected set without a dispersion point. (Fund. Math. vol. 29 (1937) p. 123.) 
Therefore $C_{\delta} \cap \sum_{\lambda<\delta} \sum_{r=1}^{\infty} J_{r}^{\lambda}$ is of first category on $C_{\delta}$, and thus there are $c$ points of $C$ not belonging to $\sum_{\lambda<\delta} \sum_{r=1}^{\infty} J_{r}^{\lambda}$; therefore we can choose an infinite $P_{\delta} \subset C_{\delta}, P_{\delta} \cap \sum_{\lambda<\delta} \sum_{r=1}^{\infty} J_{r}^{\lambda}=0$ without difficulty. Next consider the smallest $\alpha$ such that $D_{\alpha} \subset \Delta+\sum_{\beta \leqq \delta} P_{\beta}$ and $D_{\alpha}$ has not yet been dealt with in any of the previous steps. We then construct by our lemma countably many closed Jordan curves $J_{r}^{\delta}$ such that $D_{\alpha} \cap J_{r}^{\delta}$ is dense on $J_{r}^{\delta}, J_{r}^{\delta} \cap\left(\Delta+\sum_{\beta<\delta} P_{\beta}-D_{\alpha}\right)=0$, and any two points $p$ and $q$ can be separated by some $J_{r}^{\delta}$. This completes the $\delta$ th step. Thus our construction can be carried out through all ordinals of the second number class and will exhaust all the $C_{\alpha}$ 's and $D_{\alpha}$ 's. Consider now the set $A=\Delta+\sum_{\beta} P_{\beta}$. We claim that no finite set disconnects it and that any countable dense set totally disconnects it. First of all every cut of the plane intersects our set in an infinite set, thus no finite set can disconnect it. On the other hand let $D_{\alpha}$ be some countable dense set of $A$ (clearly $A$ is dense in the plane). At some step in our construction we had to deal with $D_{\alpha}$ and it is clear that $\Delta+\sum_{\beta} P_{\beta}-D_{\alpha}$ is totally disconnected, since if $p$ and $q$ are any two points there exists a $J_{r}$ separating them and $\left(\Delta+\sum_{\beta<\alpha} P_{\beta}-D_{\alpha}\right) \cap J_{r}$ $=0$. q.e.d.

By a slight modification of Miller's ${ }^{8}$ construction of a biconnected set without a dispersion point, we can construct a connected set $A$ dense in an indecomposable continuum, such that there exists an open set $O$ of the plane with $O \cap A$ having power $c$ and if $B$ is any connected subset of $A$ then $A-B$ is nowhere dense in $O \cap A$. Clearly such a set is biconnected and in fact it can be made to have no dispersion point. By a further slight modification of Miller's construction we can construct a biconnected set without a dispersion point such that if $A_{1}, A_{2}, \cdots$ is any countable collection of connected subsets of $A$, then $\cup_{i}\left(A-A_{i}\right) \neq A$ or $\bigcap_{i} A_{i} \neq 0$.

I can not decide the question whether there exists a connected set such that the complement of every connected subset of it is nowhere dense in it.

The following problems may be of some interest: Is it true that every connected set contains a connected subset not homeomorphic to it? (Points do not count as connected sets.) Every known connected set (which is subset of a Euclidean space) contains at least three different types of connected subsets. The number three can not be improved as is shown by the arc.

If a topological space does not satisfy any separation axioms the following example communicated to me by $\mathrm{S}$. Eilenberg shows that the above conjecture is not true: Let the space be the integers, the

${ }^{8}$ Miller, ibid. 
closed sets are the finite sets. Every infinite set is connected and they are clearly all homeomorphic.

Is it true that every connected set of dimension $n$ contains a connected subset of dimension $n-1$ ?

Is it true that every connected set of dimension greater than 1 contains $2^{c}$ connected subsets?

Perhaps the following theorem which A. Stone and I proved might be of some interest: Let $A$ be a totally disconnected set of power $m$ (in a separable space), then if $m$ is not the sum of countably many smaller cardinals, $A$ can be written as the sum of two separated sets of power $m$. If $m$ is the sum of $\boldsymbol{\aleph}_{0}$ smaller cardinals the theorem is in general false.

Proof. Suppose first $m \neq \sum_{k=1}^{\infty} m_{k}, m_{k}<m$. Denote by $A^{\prime}$ the set of those points of $A$ for which every neighborhood contains $m$ points of $A$. Now $A-A^{\prime}$ has power less than $m$, since by Lindelöf's theorem $A-A^{\prime}$ can be covered by countably many open sets each of which contains less than $m$ points, thus $A-A^{\prime}$ also contains less than $m$ points. Since $A$ is totally disconnected $A^{\prime}=U+V$, where $U$ and $V$ are open-closed sets. Thus both of them have power $m$. Let $p \in A-A^{\prime}$, the distance of $p$ from $U$ be $f(p)$, from $V, \phi(p), p \in U_{\alpha}$ if $f(p) \leqq \alpha \phi(p), p \in V_{\alpha}$ if $f(p) \geqq \alpha \phi(p)$. Since the power of $A-A^{\prime}$ is less than $m \leqq c$ there exists an $\alpha$ such that $U_{\alpha} \cap V_{\alpha}=0$. But then $U+U_{\alpha}$ and $V+V_{\alpha}$ are two separated sets of power $m$ whose sum is $A$; this completes the proof.

If $m=\sum_{k=1}^{\infty} m_{k}, m_{k}<m$, we define $A$ as follows: $A$ contains $m_{k}$ points in $(1 / k, 1 / k+1), 0$ belongs to $A$. Clearly $A$ is totally disconnected (its power is less than $c$, since $c$ is not the sum of $m$ smaller cardinals). Obviously $A$ is not the sum of two separated sets of power $m$.

PuRdue UnIVERSITY 